\title{
A Typology of Business Process Standardization Strategies
}

\author{
Kanika Goel • Wasana Bandara · Guy Gable
}

Received: 30 October 2019/Accepted: 19 February 2021 / Published online: 23 March 2021

(c) Springer Fachmedien Wiesbaden GmbH, ein Teil von Springer Nature 2021

\begin{abstract}
Business Process Standardization (BPS) is a strategy for improved efficiency and effectiveness of business processes. However, BPS approaches are known to vary much in practice, can consume inordinate time and resources, and are ill-understood. This study applies an exploratory analysis of BPS literature to identify alternative BPS strategies. The analysis identified three key decision-points when strategizing: (i) Approach to standardization (Bottom-up or Top-down), (ii) Choice of Master Process (Internal Exemplar, Internal Best-of-Breed, or External Exemplar), and (iii) Optimization of the Master Process (Yes or No). These alternative choices, in combination, yield 12 BPS strategies, which are described herein and instantiated by mapping 21 published BPS cases against the 12 strategy types. The resulting typology of BPS strategies can serve as a useful tool for researchers investigating BPS and may provide insight for practitioners when considering an appropriate BPS strategy, or in better understanding their existing implicit or explicit strategy.
\end{abstract}

Accepted after four revisions by Jörg Becker.

Supplementary Information The online version contains supplementary material available at https://doi.org/10.1007/s12599021-00693-0.

K. Goel $(\bowtie) \cdot$ W. Bandara · G. Gable

Queensland University of Technology,

GPO Box 2434, Brisbane, QLD 4001, Australia

e-mail: k.goel@qut.edu.au

W. Bandara

e-mail: w.bandara@qut.edu.au

G. Gable

e-mail: g.gable@qut.edu.au
Keywords Business process standardization - Typology · Strategies · Qualitative data analysis

\section{Introduction}

Business Process Standardization (BPS) is a mechanism for ensuring consistency across underlying processes, thereby supporting service-delivery excellence, and optimizing costs and benefits. ${ }^{1}$ BPS is integral to process management practices (Schafermeyer et al. 2010) and is known to positively impact business performance (Muenstermann 2015; Wullenweber and Weitzel 2007), as evidenced in time, cost, and quality metrics (Muenstermann et al. 2010b). It can also facilitate streamlining, automating, or outsourcing business processes (Rosenkranz et al. 2010). Globally, both private (e.g., Ganly 2019; Microsoft 2008) and public sector organizations (e.g., Ji and Pultz 2016) are making substantial investments in BPS.

Standardized business processes are beneficial for innovative, in-demand digital transformations such as robotic process automation (RPA), where BPS assists in improving the quality and overall efficiency of routine tasks, which in turn allows scalable RPA implementations (Aguirre and Rodriguez 2017; Asatiani and Penttinen 2016; Leshob et al. 2018). BPS also facilitates optimized execution of business processes such as smart contracts in blockchains (García-Bañuelos et al. 2017; Mendling et al. 2018). Standardized business processes are also valued in the emerging sub-discipline of business process

\footnotetext{
1 Although BPS can be seen as an overarching process management approach, in this paper when we refer to BPS, we refer to the standardization of specific processes. This is described further in Sect. 2, where we define the concept and related contexts.
} 
management-process mining-where a standard process can be used to visualize deviations between the real and expected (or standard) behaviour of business processes, e.g., for online conformance checking or compliance monitoring (Becker and Buchkremer 2019; Burattin et al. 2018; Munoz-Gama 2016). Further, business process standardization (BPS) has demonstrated a positive impact on outsourcing activities (Wullenweber and Weitzel 2007), now integral to business worldwide and to society (for example, Goel (2018) discusses the role of BPS to support with services that enable financial inclusion).

Scholars perceive BPS as an important yet under-researched topic that demands attention (Afflerbach et al. 2016; Muenstermann 2015). Notably lacking is attention to BPS strategy, i.e., the combination of key decisions an organization makes when implementing BPS, and the rationale for those choices. An improved conceptualization of BPS is needed to enhance understanding of BPS strategies and to enhance BPS design, implementation, and decision-making. To contribute to this needed deeper understanding of BPS strategies, this study addresses the question, "How can we classify business process standardization strategies that occur in practice?" In order to answer this research question, we conducted 'narrative' and 'theoretical' literature reviews (as per review genres of Paré et al. 2015), yielding a typology of 12 BPS strategies. This typology is empirically instantiated by mapping 21 published BPS case studies against the 12 strategy types.

Typologies provide a useful framework to explain outcomes (Doty and Glick 1994). They aid analysis and provide a means for comparing classes of a phenomenon (Gregor 2006), which provides the basis for theorizing and understanding the diverse contexts within which the phenomena exist. A typology of BPS strategies can also help clarify which strategy is suitable in a particular context (Collier et al. 2012).

The contributions of this paper are threefold. The resultant typology (i) provides a theory (analytic theory) for clearer thinking about the concept of BPS as well as a lexicon intended to promote discourse about BPS, (ii) assists practitioners to identify an appropriate BPS strategy considering their organizational context, and (iii) points the way to important areas of future research.

This paper is structured as follows. Section 2 presents an introductory background, followed by Sect. 3, which explains the three-phase research approach. Section 4 details the study findings from each of the 3 phases, presenting the typology and supporting evidence on how it progressively evolved. Section 5 provides a rich discussion and a research agenda based on observations stemming from the analysis. The paper concludes with a summary in Sect. 6.

\section{Background}

To clarify the concept of Business Process Standardization, we must first specify several related terms. A business process is commonly defined as " a set of logically related tasks performed to achieve a defined business outcome" (Davenport and Short 1990). Muenstermann and Weitzel (2008, p. 3) identify four sub-dimensions of a business process: (i) activities - the main actions in a process, (ii) workflow - refers to the linking of activities, (iii) resources - agents that are used for adding value to activities, and (iv) entities - "objects processed by resources." Business Process Standardization refers to the alignment of different business process variants towards a defined standard process (Muenstermann et al. 2010b). "A process variant is an observed or documented business process with a specific variation of at least one of the elements (inputs, outputs, enablers, guides and sequence of activities) for a defined part of the overall process" (Zellner et al. 2015, p. 4131). According to ISO (1996, p. 1), "Standards are documents, established by consensus and approved by a recognized body that provide, for common and repeated use, rules, guidelines or characteristics for activities or their results, aimed at the achievement of the optimum degree of order in a given context. Standardisation is defined as the activity of diffusing and adopting a standard."

The objective of BPS is to specify transparent and uniform process activities across the organization to enable organizations to achieve business process goals optimally in relation to time, cost, and quality (Muenstermann et al. 2010a). Clear, standardized processes are of better quality and devoid of repetitive and redundant tasks, which are requirements for most digital transformation initiatives. The main challenge during BPS is the transformation of existing process variants into standard operating procedures to be followed by all actors in an organization (Schäfermeyer et al. 2012). BPS requires an organizationwide effort, which involves the use of business process models or diagrams and technology. A considerable amount of time, people, and money is required to standardize a process in an organization (Afflerbach et al. 2016; Beimborn et al. 2009; Schafermeyer et al. 2010). Thus, it is essential to have a clear understanding of the process of BPS and its inherent choices. Such understanding enables a better comprehension of the concept, which is currently lacking in the field of BPS and called for (e.g., Goel and Bandara 2016; Muenstermann 2015).

BPS is a complex phenomenon that relates to other concepts (Goel and Bandara 2016), such as 'process harmonization' (Romero et al. 2015), 'process integration' (Narayanan et al. 2011), and 'process orientation' (Seethamraju and Seethamraju 2009). Furthermore, several BPM related concepts are used in the process of BPS. For 
example, modelling languages such as $\mathrm{BPMN}^{2}$ can assist in the documentation of a business process (Dumas et al. 2013). And 'process variants' can assist in identifying and managing alternatives of a business process. Though related, these are different to BPS, and in this paper, we maintain a specific focus solely on BPS for feasibility and clarity. These concepts and related literature are referred to in the process of conceptualizing BPS as deemed relevant, allowing readers to understand other related literature they might refer to when standardizing their business processes.

\section{Research Approach}

The study design had three phases (see Fig. 1), all literature-based, as detailed below. A systematic approach (following the guidelines of Bandara et al. (2015)) was used to collate literature on BPS, which formed the core input to all three phases. Information pertaining to paper extraction in the systematic literature review (including search strategies, the scope of the search, and the quality of the extracted papers, etc.) is detailed in Appendix A (available online via http://link.springer.com).

A coding rule book (following Saldana 2012) detailing how the data was captured, stored, updated, and analyzed across all three phases, was designed and applied. NVivo 11.0 was used as a tool to support the analysis. Coding occurred in multiple rounds within each phase. Two independent coders analyzed the data, and coder-corroboration sessions (to resolve any discrepancies) took place during and/or at the end of each coding round.

Phase 1 comprised a 'narrative' ${ }^{3}$ literature review, which synthesized existing research related to BPS. The objective in Phase 1 was to understand how the 'process,' or the conduct of BPS has been represented in literature, essentially to identify the stages of BPS. In the first instance, we read through the papers capturing any direct or indirect mention of indicative steps (stages) in standardizing business processes. Next, we read through all content already extracted to gain a broad understanding of what has been written about the process of BPS. The 54 BPS papers extracted formed the main input to this phase, which was supplemented with other papers outside the sample (other papers deemed relevant to explain and define new concepts that emerged during the review). By the end of Phase 1, we had gained an understanding of the process of BPS and discerned six stages of BPS along with an

\footnotetext{
2 BPMN stands for 'Business Process Model and Notation' and is the de-facto process modeling standard (Chinosi and Trombetta 2012; OMG 2011).

3 A 'narrative' literature review "attempts to identify what has been written on a subject or topic” (Paré et al. 2015, p. 185).
}

explanation of what each stage is comprised (as presented in Sect. 4.1).

Phase 2 entailed a "theoretical ${ }^{4}$ literature review based on the understanding (gained in Phase 1) of how BPS was conducted. The full set of papers (54) was revisited and analyzed to identify and synthesize any key decision-points and decision-options that were deemed to have been considered during the conduct of BPS. Table B. 1 in Appendix B presents evidence of the decision-points. Subsequent to this process, we conceptualized our literature-based typology (as illustrated in Sects. 4.2 and 4.3).

Phase 3 provided further empirical support by mapping (21) published BPS case studies against the evolving typology. These cases were a subset of the 54 BPS papers wherein empirical cases were reported. The aim was (see Sect. 4.3) to instantiate the typology, provide illustrative examples pertaining to the different types represented in the typology, and further re-specify the typology (where additional evidence suggested changes). The content of the 21 extracted case studies was analyzed using a hybrid coding approach (following Fereday and Muir-Cochrane 2008), employing the decision-points and related options discerned in Phase 2 as the a-priori coding scheme. Deductive coding was used to map the case evidence to the literature-based a-priori typology to provide empirical support. Inductive analysis captured insights not pertaining to the a-priori categories but were found to be relevant to the purpose of this study. The coding of Phase 3 proceeded in three rounds. In Round 1, any direct or indirect mentions of the decision-points and options were identified and coded to the a-priori categories. In Round 2, the content coded in Round 1 was revisited to confirm that the explicit and/or implicit meaning of content coded under a specific category belonged there. Detailed notes were used to maintain a trail of justification. Round 3 revisited the content coded inductively, seeking to identify any new themes (decision-points or options) that were not identified earlier or to populate further, or to elaborate on the existing categories.

\section{A Typology of Business Process Standardization Strategies}

\subsection{Study Phase 1: Understanding of the Process of Business Process Standardization}

A narrative literature review was performed to develop a clearer understanding of how Business Process Standardization (BPS) is conducted. In particular, we sought to

\footnotetext{
${ }^{4}$ A 'theoretical' review, "draws on existing conceptual and empirical studies to provide a context for identifying, describing, and transforming into a higher order of theoretical structure and various concepts, constructs or relationships" (Paré et al., 2015, p. 188).
} 
Fig. 1 Overall study design

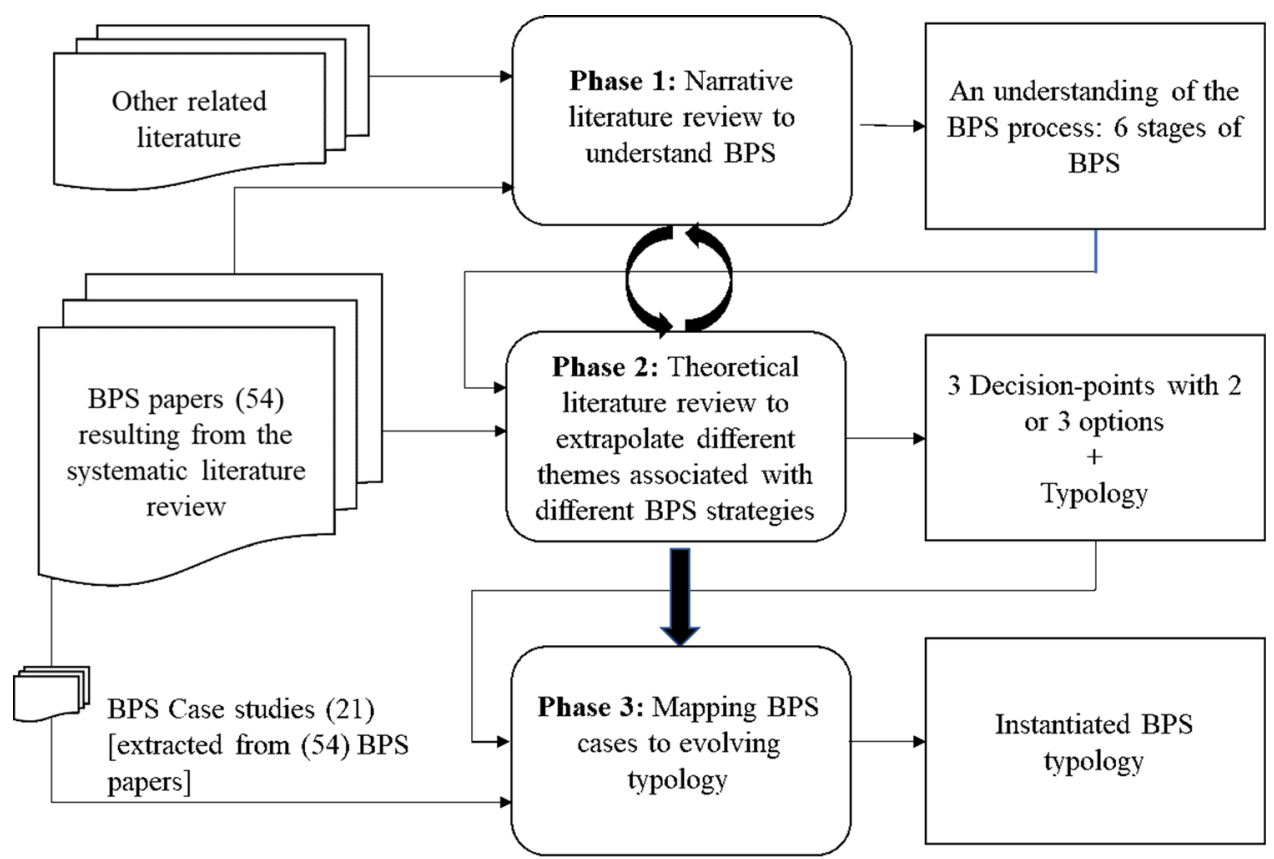

understand BPS and to succinctly identify the main stages of a BPS initiative in order to later (in Phase 2) delineate the key decision-points (and their options) during the process of business process standardization. BPS initiative refers to the conduct or process of business process standardization in an organization. A review of the literature revealed that for BPS to happen, some form of a trigger is essential. This trigger could be a decision made by top management (e.g., stimulated by observed process inefficiencies) or could be a decision by employees who, of their own accord, seek consistency through standardization (e.g., to overcome daily issues affecting their productivity and job satisfaction) (Kwon 2008). Our synthesis of the literature indicated that irrespective of the reason for BPS initiation, the following seven stages of BPS are evidenced.

Stage 1 - Documentation: This stage involves documenting details of variants (single or multiple) of the process to be standardized (Muenstermann and Eckhardt 2009; Muenstermann and Weitzel 2008; von Stetten et al. 2008; Ungan 2006). The details of already documented variants need to be checked to ensure all specifications of the process are adequate. Process models can be documented using flowcharts (Ungan 2006), process modeling language (e.g., BPMN), or detailed written descriptions.

Stage 2 - Modularization: The next stage is to modularize the variants, i.e., to divide them into meaningful, coherent parts (Muenstermann and Weitzel 2008; von Stetten et al. 2008). This is to ensure that the necessary aspects of the process are captured to the required level of detail (such as who is responsible for tasks and who is accountable for the execution of the various parts of the process) (von Stetten et al. 2008), while also managing the associated complexities of maintaining such details.

Stage 3 - Derivation of Master Process: The next important stage is to derive a 'point of reference,' which we refer to as the 'Master' process (Muenstermann and Weitzel 2008) that will be used to standardize the processes. Note that some authors refer to this as the 'archetype process' (Muenstermann and Eckhardt 2009). A Master Process is essentially a business process reference model (vom Brocke 2007), a process that is reusable, displays exemplary practices, and has widespread applicability (Rehse et al. 2017). A Master Process can be derived either from within the organization (an internal process) or from a process or set of processes external to the organization.

When choosing an internal process, there are two options. First, on an investigation of the documented variants, the organization may choose an entire process that it considers to be best practice and against which it desires to standardize the other processes (von Stetten et al. 2008). We refer to this approach as choosing an 'Internal Exemplar' as the Master Process. Fettke et al. (2005) propose a framework to describe business process reference models, which can assist in selecting an appropriate internal Master Process. Alternatively, the organization may find modules of different process variants to be efficient and decide to amalgamate these modules to form a new process that consolidates internal best practices (Muenstermann et al. 2010b), also referred to as 'inductive reference modeling' (Rehse et al. 2016; Scholta et al. 2019). We refer to this approach as the 'Internal Best-of-Breed' Master Process. 
Literature presents multiple approaches to obtaining inductive reference models (e.g., Gottschalk et al. 2008; Li et al. 2010; Scholta et al. 2019), which can be adopted to derive an 'Internal Best-of-Breed' Master process.

Additionally, organizations may also choose to standardize their processes against a process external to the organization (Kauffman and Tsai 2010). For example, when an organization must abide by a standard external process (e.g., Sarbanes-Oxley Act (Romano 2004)) or is aware of a best practice external process or practices (e.g., Information Technology Infrastructure Library (ITIL) (AXELOS 2020), or Supply Chain Operations Reference (SCOR) (Bolstorff and Rosenbaum 2007)). We refer to this as an 'External Exemplar.' The literature (e.g., Houy et al. 2011; Winter et al. 2009) indicates that established process reference models may differ from the needs of the organization (given their generalized intentions), which suggests that adoption of an external process reference model requires careful consideration of an organization's needs and demands.

Stage 4 - Isolation of specificities: In this stage, the specificities of the Master Process that cannot be replicated to all process variants need to be isolated. Manrodt and Vitasek (2004) suggest a standard process should be macro-level in nature such that some level of customization across instances is allowed and at times needed. Some variants of the process may have associated specificities that may not be appropriate for other variants, e.g., for contextual reasons, such as local legislation. Therefore, if the Master Process chosen has such specificities, they need to be sequestered and dealt with in isolation while other parts of the process will conform to the Master process and have no specificities (Muenstermann and Weitzel 2008; von Stetten et al. 2008). Isolation of specificities can dramatically reduce the number of different steps involved in a Master process. On introducing these changes, an enhanced version of the Master Process is obtained.

Stage 5 - Optimization: The Master Process chosen in Stage 3 may be Optimized (improved) by various means, including analyzing information about competitors, using experts, looking at external reference process models for insights for best/common practice, and using information consultants (von Stetten et al. 2008). However, the decision as to whether to optimize will depend on diverse organizational factors.

For example, the goal of the process standardization effort (Muenstermann and Weitzel 2008; von Stetten et al. 2008; Ungan 2006) is pertinent. To elaborate, one organization might want to standardize its sales process and like that process to be the 'best possible' method of execution (something with which they distinguish their firm). On the other hand, another organization may more simply seek an 'adequate' sales process (thereby constraining their investment) standardized across the organization. Available resources, process management capacity, and overall process standardization approach play a role in an organization's decision on, if, and when to consider optimization (Muenstermann et al. 2010b; Rahimi et al. 2016). The extent to which external best practices are available and accessible for the given process domain can also influence the decision to optimize. To elaborate, if an organization considers an external standard to be best practice, the Master Process may be enhanced in accordance with that external standard (Dai et al. 2011). This would be particularly true for organizations such as banks, which seek to standardize their process according to nation-wide or sector-based external standards.

Stage 6 - Approval by formal body: Once the final Master Process is derived, it needs to be approved and governed by a formal body (ISO 1996; Nesheim 2011; Rosenkranz et al. 2010) for it to meet the definition of a standard. The formal body can be the top management of the firm, an external body, or some other higher authority whose approval or governance is required for the functioning of the organization (Nesheim 2011). This is true whether standardization efforts are initiated by management or by employees. When management initiates BPS, it will mandate the standard in the organization. In the case of an employees' initiative, the employees would have worked together to discern a standard process, which still needs to be approved by a formal body.

Stage 7 - Unification: When implementing the standard, the process variants are unified with the defined standard. Unification refers to the activity of aligning existing variants of a process against a standard process, where consideration is given to various factors such as data, people, and technology (Romero et al. 2015). When standardizing multiple variants, challenges regarding differing mindsets, skills, data formats, and attitudes (e.g., acceptance of technology) may have to be confronted and addressed. Zellner et al. (2015) presented one approach to unify process variants. Further research in the domain of process variant management can uncover other ways of unifying process variants. Unification is also referred to as 'homogenization' (Muenstermann et al. 2010a; Muenstermann and Weitzel 2008; von Stetten et al. 2008).

\subsection{Study Phase 2: A Preliminary Typology \\ of Business Process Standardization}

As explained in Sect. 3 (and Appendix A), all 54 papers resulting from the systematic literature review were examined to identify decision-points. The BPS stages extracted in Phase 1 were employed as a 'lens' to identify different decision-points while remaining open to others beyond the BPS stages. The decisions, at times, were 
explicitly mentioned in the papers. At other times they were only implied. For example, decision-points were implied through the different scenarios observed for a given stage. To illustrate, we found statements like "Future [company] did not incorporate external recruiting process excellence" (von Stetten et al. 2008, p. 5) and "Dream [company] then incorporated externally available business process excellence into the archetype process A" (Muenstermann and Weitzel 2008, p. 14) under the stage Optimization of Master Process. Thus, in the latter scenario, excellence was sought through Optimization. In the first scenario, the firm was content to satisfice. From statements like these, it was inferred that an organization may or may not choose to optimize the Master Process.

Three decision-points (D1-D3) with two, three, and two options respectively were discerned during this phase, thus providing the basis for the typology. Table B. 1 in Appendix B presents the supporting evidence for the decision-points and the options identified. Each of the three decision-points and the related options is outlined in more detail below. The typology was formed to represent the alternative combinations of the decision-options, yielding 12 combinations $(2 \times 3 \times 2)$ or 12 alternative BPS strategies (see Table 1). The 12 strategies are described in Sect. 4.3 (where case examples are mapped to them).

Decision-Point 1 (D1) - BPS Approach: This refers to how the BPS initiative emerges. Given the magnitude of this first decision-point, we refer to it as the BPS Approach. A BPS approach can be either: Top-down or Bottom-up. Top-down standardization occurs when standardization is enforced by authorities (i.e., mandated by management) (Kwon 2008). Top-down standardization is normative and specifies how things need to be done. These are formal projects initiated by upper management.

Consider an alternative scenario where management has no intention to drive or guide standardization and is unaware of bottom-up, organic standardization being driven by employees. In this case, even though BPS may be occurring in the background, these standardization efforts remain 'invisible' to management, thus, are not an issue of management consideration. That said, if management sees value in BPS, it behooves them to be aware of such employee actions pertaining to any standardization efforts. Where management is aware of Bottom-up standardization, they can either sanction it or not, explicitly or implicitly. Where management sees or anticipates value from perceived Bottom-up standardization, they may simply adopt a watch-and-see approach. Alternatively, they may seek to promote it, for example, by offering recognition to people taking such initiatives. Though originating from an organic, employee-driven initiative, given a management decision to subsequently orchestrate such a standardization effort, what was Bottom-up, becomes a Top-down approach.

This Top-down vs. Bottom-up perspective helps management to understand the different means that BPS can emerge from and guide management to adopt appropriate action to support or curtail BPS.

Decision-Point 2 (D2) Type of Master Process: When standardizing a process, a Master Process is first sought before the process is standardized (see Stage 3 of Sect. 4.1 for further details). This decision-point relates to the type of Master Process chosen and has three options: Internal Exemplar, Internal Best-of-breed, or External Exemplar.

Summarily, an Internal Exemplar refers to a process that is chosen from within the organization as a point of reference, a preferred complete end-to-end single process. Alternatively, an amalgamation of modules from internal variants of the process can be chosen, which is referred to as an Internal Best-of-Breed Master Process. Lastly, the organization can choose to standardize its processes against an external Master Process. The external Master Process can be another company's, or an external reference process model that captures best/ recommended practice (e.g., SCOR), or an external reference process model resulting from legislation (e.g., Sarbanes Oxley). The Master Process selection can also depend on the organization's size, maturity, and experience.

Decision-Point 3 (D3) Optimization of Master Process: This decision-point occurs at Stage 5 (see Sect. 4.1) and relates to whether the firm invests in further modifying the Master Process to reflect best practices or not. Such best practices can be sourced from external or internal consultants or experts, from what has been observed in the past that works well in the organization, from external reference process models, etc. The degree of adaptation can vary much depending on the process, the starting point of Optimization, and the company context. The literature suggests that such refinement of the Master Process and related investment depends on the organization's goals with BPS (e.g., excellence vs. satisficing). An organization may only be interested in having a standard routine process throughout the organization (von Stetten et al. 2008) or may want to improve its Master Process by integrating knowledge from external or internal consultants, analyzing competitors (Muenstermann et al. 2010b), and then standardize the variants against the improved Master Process. These goals will be influenced by other contextual aspects such as resource constraints (e.g., does the organization have resources to improve a process once its standardized (Rahimi et al. 2016)), methodological choices (e.g., a staged approach to process improvement where one first gets the processes standardized, then shifts to continuous improvement plans (Muenstermann et al. 2010b)), or feasibility. 
Table 1 Strategies of business process standardization

\begin{tabular}{|c|c|c|c|c|c|}
\hline \multicolumn{3}{|c|}{ Decision-points and options } & \multirow[b]{2}{*}{ Strategy } & \multicolumn{2}{|l|}{ Case instantiations } \\
\hline $\begin{array}{l}\text { (D1) } \\
\text { Approach }\end{array}$ & $\begin{array}{l}\text { (D2) } \\
\text { Type of } \\
\text { master } \\
\text { process }\end{array}$ & $\begin{array}{l}\text { (D3) } \\
\text { Optimization of } \\
\text { master process }\end{array}$ & & Source & \# \\
\hline \multirow[t]{7}{*}{$\begin{array}{l}\text { Top- } \\
\text { down }\end{array}$} & \multirow[t]{2}{*}{$\begin{array}{l}\text { Internal } \\
\text { exemplar }\end{array}$} & Yes & 1 & $\begin{array}{l}\text { Agnar et al. (2004); Kwon (2008); Manrodt and Vitasek (2004); Muenstermann } \\
\text { et al. (2009); Muenstermann et al. (2010a); Rahimi et al. (2016, Case 3); } \\
\text { Rosenkranz et al. (2010, Case 2); Schafermeyer et al. (2010, Case 2) }\end{array}$ & 8 \\
\hline & & No & 2 & $\begin{array}{l}\text { Afflerbach et al. (2016); Rosenkranz et al. (2010, Case 3); von Stetten et al. } \\
\text { (2008) }\end{array}$ & 3 \\
\hline & Internal & Yes & 3 & Muenstermann et al. (2010b); Muenstermann and Weitzel (2008) & 2 \\
\hline & $\begin{array}{l}\text { best-of- } \\
\text { breed }\end{array}$ & No & 4 & Kettenbohrer et al. (2013a, b); Rahimi et al. (2016, Case 2) & 3 \\
\hline & External & Yes & 5 & van Wessel et al. (2006) & 1 \\
\hline & exemplar & No & 6 & N/A & \\
\hline & & & & Sub-total: & 17 \\
\hline \multirow{7}{*}{$\begin{array}{l}\text { Bottom- } \\
\text { up }\end{array}$} & \multirow{4}{*}{$\begin{array}{l}\text { Internal } \\
\text { exemplar } \\
\text { Internal } \\
\text { best-of- } \\
\text { breed }\end{array}$} & Yes & 7 & Roubert et al. (2016) & 1 \\
\hline & & No & 8 & Rahimi et al. (2016, Case 1); Schafermeyer et al. (2010, Case 1) & 2 \\
\hline & & Yes & 9 & N/A & \\
\hline & & No & 10 & N/A & \\
\hline & \multirow{3}{*}{$\begin{array}{l}\text { External } \\
\text { exemplar }\end{array}$} & Yes & 11 & Kauffman and Tsai (2010) & 1 \\
\hline & & No & 12 & N/A & \\
\hline & & & & Sub-total: & 4 \\
\hline
\end{tabular}

\subsection{Study Phase 3: Instantiation of Different Strategies of Business Process Standardization}

The BPS strategies typology ${ }^{5}$ is based on the three decision-points and their options, as explained above, yielding 12 choice combinations $(2 \times 3 \times 2)$ or 12 alternative BPS strategies. These 12 types were instantiated with published BPS case studies, as presented in Table 1. We analyze the pattern of strategies (combinations of the three decisions) observed, suggesting criteria that might influence choices. As mentioned in Sect. 3, the coding design in this phase was hybrid. Although the 12 types and their related decision-points and options provided the deductive coding framework, we were open to identifying new decisionpoints or options from the cases. Still, none emerged from this pool of cases.

The 21 cases identified from this review aligned with eight of the 12 strategies; four strategies were not instantiated (Strategies 6, 9, 10, and 12). Each strategy is

\footnotetext{
5 Unlike a taxonomy, a typology does not provide decision rules for classifying, but rather identifies multiple ideal types, each of which represents a unique combination of the pertinent attributes that are believed to determine the relevant outcome(s) (Doty and Glick, 1994).
}

explained, drawing from the case examples. For those that were not instantiated, we provide fictitious case scenarios to demonstrate the strategy's potential relevance. We present each strategy next, grouping them primarily according to D1 (the BPS approach) and secondarily based on their instantiation (or not) with case studies.

\subsection{Strategies with a Top-down Approach}

\section{Instantiated Top-down Strategies}

All but one of the six top-down strategies was instantiated.

Strategy 1: Top-down standardization, with an Internal Exemplar Master Process and Optimization of Master Process

Strategy 1 was instantiated by the most cases (a total of eight). This strategy is employed by organizations where BPS is the result of formal authority and where standardization efforts are related to continuous improvement.

For example, the organization 'VISION' (Muenstermann et al. 2010a) launched a project to standardize its recruitment process across all its autonomous divisions. VISION pre-selected the headquarters' recruiting process as the Internal Exemplar Master Process and then 
optimized it, using insights gained from 3 large competing organizations. This helped VISION standardize its processes against an Internal Exemplar Process enhanced with industry-identified best practices, assisting the organization in improving its recruitment process.

Strategy 2: Top-down standardization, with an Internal Exemplar Master Process and no Optimization of the Master Process

Strategy 2 was instantiated by three case studies and is employed by organizations where the process standardization efforts are not driven by any continuous improvement initiative, and the focus is on the consistency of processes based on existing/current internal practices. This strategy of BPS is common with the rise of globalization and the need for greater consistency in services/operations (Afflerbach et al. 2016).

For example, 'Future' (von Stetten et al. 2008) has three autonomous divisions, each responsible for their own results and using different information systems to recruit staff. 'Future' launched a standardization project (Topdown) in 2004 to achieve consistency in the recruitment process across all divisions. The priority was to achieve maximum internal and external transparency without emphasizing further process improvement. Since the 'Future' processes were mature, the headquarter's process was chosen as the Internal Exemplar Master Process against which other processes were standardized.

Strategy 3: Top-down standardization, with an Internal Best-of-Breed Master Process and Optimization of Master Process

Strategy 3 was instantiated by two cases and employed by organizations with multiple variants of processes, but where no single variant is an obvious candidate for Internal Exemplar Master Process (as in Strategy 1). It is a strategy for organizations that are focused on continuous process improvement (beyond mere process consistency). Organizations undertaking this form of BPS are likely to have all the process variants documented (e.g., as a part of normal formal procedures) and thus will recognize pockets of excellence in different variants. Therefore, they are willing and able to merge best practices from parts of different processes to obtain an Internal 'Best-of-Breed' Master Process.

In the case study outlined by Muenstermann and Weitzel (2008), a multinational firm, 'Dream,' had several process variants and launched a BPS program to reduce costs and progress continuous improvement.

Strategy 4: Top-down standardization, with an Internal Best-of-Breed Master Process and no Optimization of Master Process

Strategy 4 was instantiated by four cases. Unlike Strategy 2 (which is similar), these organizations are likely to have several process variants but no single end-to-end process that can serve as an exemplar. Instead, the betterfunctioning modules of such variants are merged to obtain an Internal Best-of-Breed Master Process.

For example, the goal of Lufthansa Technik (LHT) in Germany (Kettenbohrer et al. 2013b) was to standardize the process that provides a quality assessment of suppliers and supplier-related products. There were several working variants of the same process, with modules that were considered best practice internally by the organization. Such pockets/modules were extracted and then amalgamated to derive the Master Process, which was then used for standardization.

Strategy 5: Top-down standardization, with an External Exemplar Master Process and Optimization of Master Process

Strategy 5 was instantiated by one case. BPS was formally introduced to standardize against an external best practice process while also having a strong focus on continuous improvement. Strategy 5 particularly relates to organizations that do not have in-house processes that can be used as a Master Process or are in industries in which there is a need or motivation to abide by external standards (for example, SCOR (Bolstorff and Rosenbaum 2007)) to implement minimum cross-industry supply chain standards). This is also the main difference between this strategy and Strategies 1 and 3.

Wessel et al. (2006) discuss a standardization initiative across a financial firm with over 50 divisions worldwide. The firm had implemented PeopleSoft as a system, applying the recommended vendor proposed reference models to standardize their business processes. This helped the firm to ensure that the data privacy and protection regulations were followed in the same way across all the divisions. Further, there was a focus on continuous improvement to ensure that their Human Resource policies followed best practices.

\section{Non-instantiated Top-down Strategies}

Only one of the six top-down strategies was not instantiated.

Strategy 6: Top-down standardization, with an External Exemplar Master Process and no Optimization of Master Process

Strategy 6 was not instantiated by any of the identified case studies. However, it is feasible, and further research may uncover such cases. This strategy differs from Strategy 2 and 4 in that it is suitable for organizations that do not have any internal process(es) appropriate as a reference point for standardization, so may want to standardize their processes against a best-known external process, such as the Sarbanes Oxley Act. Unlike Strategy 5, the Master Process is not optimized as it may not be perceived necessary, or the organization is expected to abide by the external reference model strictly. 
For example, fictitious 'University A' may wish to standardize its financial reporting process. To do so, the university may use the Sarbanes Oxley Act and related processes as an external reference model because of the advantages of the prescribed processes on financial disclosure and reporting. Because of the comprehensive nature of the Act, the university may not optimize it.

\subsubsection{Strategies with a Bottom-up Approach}

Only three of the six bottom-up strategies were instantiated.

\section{Instantiated Bottom-up Strategies}

Strategy 7: Bottom-up standardization, with an Internal Exemplar Master Process and Optimization of Master Process

Strategy 7 was instantiated by one case and is relevant to organizations where standard processes are required for efficiency, and people are encouraged to adhere to uniform practices across the organization (Roubert et al. 2016). Further, an organization considering this strategy would have a representative end-to-end process, leading them to use an existing Internal Exemplar Master Process and would be committed to improvements that lead to the Optimization of the Master Process as the standard. The difference between this strategy and Strategy 1 is that the standardization effort here is organic and driven by employees. In contrast, in Strategy 1, the standardization effort is mandated by the top management.

In the case study discussed by Roubert et al. (2016), employees in a nanotechnology firm realized the need for a standard process related to nanoparticle experimentation. The current set of guidelines the company used to conduct experiments became the Internal Exemplar Master Process. This was further enhanced by collating information from external protocols. Mutual consensus on integrating such best practices helped develop standard practices related to nanoparticles across the firm.

Strategy 8: Bottom-up standardization, with an Internal Exemplar Master Process and no Optimization of Master Process

Strategy 8 was instantiated by two cases. This strategy is suitable for organizations with diverse variants for the same process, which may cause inconsistencies and inefficiencies. However, a mature process variant exists, which could be a suitable as the Internal Exemplar. The employees believe that a standardized process will improve their efficiency and are willing to drive the initiative. Here, the Internal Exemplar Master Process is not optimized, making this strategy different from Strategy 7.

Case study 1 by Schafermeyer et al. (2010) describes the German telecommunication provider (TCSP) that started using off-the-shelf software as a part of its client order process, which employees liked and decided to adopt as a standard. Therefore, the standardization initiative was a result of employees' desire to have a consistent process. Inhouse software was developed to address employees' needs and achieve the desired standardization. The internal inhouse software developed served as the Internal Exemplar Master Process. Since the aim was solely to have a consistent client order process across all the locations of the organization, the Master Process (the in-house software) was not optimized with external best practices.

Strategy 11: Bottom-up standardization, with an External Exemplar Master Process and Optimization of Master Process

Strategy 11 was instantiated by only one case. In this strategy, the staff initiates the Bottom-up standardization process to improve their job performance. This type likely arises in industries where an external standard may seem more suitable or where current processes may not be adequate to become a Master Process against which variants can be standardized. The difference between this and Strategies $7-10$ is that the employees may not find an entirely internal process or an amalgamated version of variants suitable as a Master Process.

In the case study reported by Kauffman and Tsai (2010), the employees of an IT firm-initiated Bottom-up standardization to ensure consistent quality of the technology sold to the consumers. For example, software vendors aim to standardized practices to launch enterprise software solutions, so the employees chose an industry-wide-practice standard, which was then subject to continuous refinement. This assisted the IT firm to maintain consistent practices across the firm and compare their performance across the industry.

\section{Non-instantiated Bottom-up Strategies}

Strategy 9: Bottom-up standardization, with an Internal Best-of-Breed Master Process and Optimization of Master Process

Strategy 9 was not instantiated by any of the case studies. However, we argue that it is suitable for contexts where the staff involved in executing a process takes leadership and want to derive a standard process based on existing practices that have been tested within the organizational context but where no single mature-enough process exists that can be used end-to-end as the standard. Thus, they would attempt to derive a new standard based on amalgamating modules of best practices from different areas to form a new Internal Best-of-Breed Master Process. The assumption here is that the employees (i.e., the process executors) would also want continuously to improve this newly derived process. Such can only occur where there is an organizational context that is flexible and a culture that encourages and empowers employees to take initiatives. 
For example, University A may decide to advertise Ph.D. recruitment across all faculties. However, different faculties may have unique criteria and procedures for the intake of a Ph.D. student. This may confuse students, which is, in turn, communicated to the staff. To address students' concerns, staff engaged in the recruitment processes across the faculties may attempt to standardize the process of Ph.D. student recruitment. Staff may coordinate multi-faculty discussions and understand everyone's version of recruiting a Ph.D. student and pick best practices from diverse recruitment practices to derive an Internal Best-of-Breed Ph.D. student recruitment process. This may then be optimized by integrating practices recommended by external learning advisors and consultants and periodic reviews. For this strategy to be successful, the employees need to have a deep desire for continuous improvement and some degree of process-centric thinking. Furthermore, the employees should have some flexibility in their work, with rewards and recognitions to encourage them to take initiatives like this.

Strategy 10: Bottom-up standardization, with an Internal Best-of-Breed Master Process and no Optimization of Master Process

Although not instantiated by any case study, we see this strategy as a possible option. Similar to Strategy 9, if the employees' goal is simply consistency, then the Master Process first derived would not be optimized further (i.e., the standard process decided upon initially will be 'the' process, and no further optimization efforts will be executed).

Strategy 12: Bottom-up standardization, with an External Exemplar Master Process and no Optimization of Master Process

This strategy was not instantiated, but we see its potential to be relevant when those leading the BPS efforts see the External Master Process as 'the' process to follow and would be satisfied with reaching a similar process execution internally (and no further optimizations are deemed necessary at that stage).

For example, in fictitious University A, the employees may be struggling to find an optimal way to conduct online exams. Furthermore, staff may be conducting online exams differently, confusing students. This can result in dissatisfied students and other process issues (such as a breach of assessment policies). To overcome this situation, the staff may initiate the standardization of the online exam process. They may adopt the online exam process of some other university, which they believe in having better online exams and use that process to standardize their process. This strategy is like Strategy 6 except that the initiative is Bottom-up (driven by the employees) rather than being enforced by the top management.

\section{Discussion}

This paper presents a typology of business process standardization strategies, derived using an exploratory multiphased approach. The literature-based typology resulted in 12 different BPS strategies based on three key decisionpoints (D1- D3) and their related options. Analysis of 21 published case studies instantiated eight out of the 12 BPS strategies. We argue that the four strategies not instantiated are viable, as shown via a fictional case example (University A). Our analysis of the instantiated cases also enables an understanding of situations where a standardization strategy might be most relevant to assist researchers and practitioners in realizing which strategy may be suitable for different contexts.

\subsection{Discussion Around Decision-points}

We make several observations from Table 1 regarding each of the three, key decision-points. For the decision-point BPS Approach, more cases applied a Top-down Approach (17 cases across Strategies 1, 2, 3, 4, and 5, over $80 \%$ of cases) than a Bottom-up Approach (four cases across Strategy 7, 8, and 11). The case examples for Top-down often came from larger organizational contexts, where management required consistency of operations and/or was planning to use process-centric technologies that require standard processes. These were larger-scale BPS efforts, where clear Top-down sponsorship with committed resources was essential. Consultants are typically involved in such initiatives (Rahimi et al. 2016; Rosenkranz et al. 2010).

Bottom-up standardization efforts observed did not have the same resources and executive support but rather emerged organically in contexts where employees are frustrated with the inefficiencies resulting from inconsistent processes or driven to improve business processes continuously. The organic nature of Bottom-up cases is one reason we believe that the majority (three out of four) of non-instantiated strategies were Bottom-up; they are less likely to be documented. Further research into success stories, employee recognition articles, and in-depth case study research may result in an increased instantiation of Bottom-up strategies. Another reason could also be that Bottom-up studies are not reported in the standardization domain, which was the focus of this study.

For both Top-down and Bottom-up approaches, for the decision-point type of Master Process, an Internal Exemplar Master Process was the most frequently instantiated option (Strategies 1, 2, 7, and 8). Of the 21 cases, two thirds (14) adopt an Internal Exemplar, 65\% of Top-down initiatives (11 of 17), and $75 \%$ of Bottom-up initiatives (three of four). These case examples showed how 
management and/or employees preferred to use an internal end-to-end process that had proven its viability within the organizational context. It was more efficient and less risky than trying to create a new Master Process. Internal Bestof-Breed Master Process strategies (Strategies 3, 4, and 9) were the next most popular choice. All five Best-of-Breed Master Process strategy instantiations were Top-down but zero Bottom-up. The case examples for these types showed that the BPS champions considered specific modules across different process variants to be strong and chose to amalgamate them to obtain the Internal Best-of-Breed Master Process. This seemed to be the case for large organizations that have multiple variants of the same process. Interestingly, we observed only one External Exemplar choice for each of the Bottom-up and Top-down Approaches (Strategies 5 and 11). The choice of an External Master Process was found in cases where there is a need to maintain consistency with industry-wide practices (for example, a banking organization must follow industrybased rules and regulations for specific processes) or when new organizations, with immature processes, find it is more viable to use a process that has been applied in other similar contexts. Our literature-based approach may have also influenced this observation, as those BPS initiatives which choose an External Master Process are often described as compliance efforts (rather than process standardization efforts). As indicated by Sadiq et al. (2007), compliance ensures business processes are in accordance with the prescribed and standard set of norms which stem from the legislature or regulatory bodies (e.g., Sarbanes Oxley), standards and codes of practice (e.g., SCOR), and also business contracts. Hence this may have limited the identification of published resources and reduced the supporting empirical base for this type (for our search terms were scoped and limited to process standardization and did not cover process compliance as a topic area).

In both BPS approaches, Optimization of the Master Process (Strategies 1, 3, 5, 7, and 11) was more popular than no Optimization (Strategies 2, 4, and 8). Overall, 13 of the $21(62 \%)$ chose to optimize the selected Master Process (11 of 17 Top-down initiatives (75\%) and two of four Bottom-up initiatives (50\%)). Considering the frequency of Optimization in conjunction with the type of Master Process, we note that $73 \%$ of Top-down, Internal Exemplars chose to optimize (eight of 11). Organizations that have dedicated enough time and resources may decide to optimize the Master Process during the standardization initiative. Such organizations are focused on continuous improvement and desire more than just the consistency of processes. When organizations require mere consistency of processes, they may not optimize the Master Process (or postpone such to a later date). Optimization of the Master Process is an additional step in standardization, requiring extra time and resources, and hence an important decision to make.

\subsection{Other Interesting Observations}

Strategy 1 (Top-down standardization, with an Internal Exemplar Master Process and Optimization of Master Process) was found to be by far the most popular of all strategies within the pool of case examples analyzed. Given the important role that process standardization plays within diverse initiatives, it is not surprising that management sponsored enterprise-wide BPS efforts (Top-down) are prominent. Selecting an Internal Exemplar Master Process is more efficient and less risky as the process has been 'tested' within the company context. Once standardized, the process can be further enhanced through continuous improvement.

It is difficult to draw conclusions from the relatively small sample size of instantiated Bottom-up cases. However, some patterns are discernible. Three of the four Bottom-up cases chose an Internal-Exemplar Master Process. Understandably, the impetus for many such organic initiatives is the observed superiority of a particular inhouse instance of the process. It is noteworthy that the single instance of a Bottom-up External-Exemplar, related to the nature of the industry (working with nanotechnology) and chose to adopt a standard external process for experiments. We note no instances of Bottom-up, Internal Best-of-Breed. As mentioned earlier, it is likely that fewer Bottom-up cases are documented. However, this may also be due to the formal coordination required to agree on which variants form the standard. It may be that tighter control and more substantial central authority are needed to address disagreement about process variants. Therefore, we may consider that employees may rely on an external established standard when internal discrepancies are noted.

We also note that both the External Exemplar cases (one Top-down, one Bottom-up) chose to optimize. Adopting an External Exemplar as the standard involves bringing inhouse a variant that is new to the organization. Optimization is understandable, as there is a need to adapt the external process to the firm's specific expectations, culture, and context. Alternatively, where adopting something that has evolved in-house (either exemplar or best-of-breed), there is already a natural fit.

We acknowledge the 'options' that we have identified for each decision-point may not be so discrete in practice. For example, the preponderance of instances of Optimization of Internal Exemplars may represent some blending of the Internal-Best-of-Breed model into the Internal-Exemplar model, with Best-of-Breed features of variants incorporated into the initial Internal Exemplar during Optimization. Further, we have suggested earlier 
that Bottom-up initiatives, once recognized by management, may be sanctioned or even promoted and that ultimately, the standard chosen Bottom-up must be endorsed by management, thus entailing some Top-down influence. Also, a Bottom-up initiative may be taken over by management, thereby becoming Top-down.

Furthermore, once implemented, these strategies are not set-in-stone; they can evolve or change completely. For instance, an organization may choose Strategy 2, Topdown standardization with Internal Exemplar Master Process, and no Optimization of Master Process, but later decide to optimize the Master Process (hence proceed with Strategy 1: Top-down BPS with Internal Exemplar Master Process and Optimization of Master Process). This may occur when the driving priority is to have a unified approach to the same process across an organization, but later, the standardized process can be further improved. It is also likely that strategies with a Bottom-up approach become common after the conduct of Top-down approach initiatives. For instance, when the positive impact of a Topdown standardization is observed for one process, it may trigger the emergence of Bottom-up standardizations for other processes. The opposite can also be the case, where the positive impact of Bottom-up standardization is observed by company executives, and Top-down standardization for other core processes is triggered. A similar evolution is possible for the selection of the type of Master Process. An organization may choose an Internal Exemplar Master Process first, and in the next iteration of standardization may opt for an External Exemplar Master Process if they find a better external process. This shift may also result from external factors such as pressure to comply with external standards or requirements to abide by new regulations outlined by the government or the company headquarters, etc. Understanding different strategies help organizations also understand how they can phase out their standardization initiatives and work.

\subsection{Limitations and Future Work}

We acknowledge certain limitations in the paper. The construction of the typology was literature-based and thus is vulnerable to the common limitations of solely literaturebased analysis. Although we employed a rigorous search procedure and coding process for retrieval and analysis of relevant papers, the decisions-points forming the typology are limited to what is reported in the literature, and future primary data collection may identify other key decisionspoints. Similarly, the instantiation of the typology was limited to the 21 published cases (from 18 papers). Regarding the coding approach, one may critique the approach applied in Phase 2 for the inductive derivation of the decision-points and their options, particularly concerning the transparency of this process. We have addressed this by articulating the paper extraction and coding process and with further arguments about how we see these core decision-points in the BPS process as the main determinants contributing to the different strategies of BPS. Also, we do not claim that the decision-points identified here are the only ones that characterize BPS. They were identified in the current literature and instantiated by the extracted cases. Although the coding process was open to identifying new decision-points or options, the extracted cases did not point to any new decision-points or options. This also means that the decision-points and options here are limited to what the current literature implicitly or explicitly mentions, but more may exist in practice. Hence, we call for further research to uncover other examples and to investigate these BPS strategies further.

The work presented in this paper points to opportunities for future research. See Enumeration 1 below for a list of proposed future research questions (F-RQs) stemming from this work. BPS is still an under-researched area with a limited understanding of the BPS concept itself (Goel and Bandara 2016; Muenstermann 2015). This may explain why some of the proposed strategies were not instantiated by the published cases. It also raises the possibility that the full range of BPS strategies may not yet have been identified. We suggest that future researchers might build upon the presented typology of BPS strategies, identifying other decision-points and options (F_RQ1). Given that BPS decision-points depend on context, researchers may also explore the organizational requirements and diverse contexts (e.g., process and project characteristics, target outcomes, critical success factors, etc.) that relate to each BPS strategy (F_RQ2). We also propose future research towards developing a deeper understanding of what capabilities and resources are needed for the different strategies to be operationalized and how to best obtain them (F_RQ3). We briefly discussed the potential evolution of BPS strategies, where companies can move from one type of strategy to another, and this can be further investigated to more clearly understand when particular strategies are most appropriate (in terms of timing, available resources, and also situational contexts), especially within an evolutionary BPS program across an organization (F_RQ4). Future researchers may also explore the impact of each strategy on an organization (F_RQ5), enabling practitioners to have an enhanced understanding of the implications of each strategy. Finally, the work presented in this paper was exploratory and based on secondary data-published literature. Future research based on primary data (such as expert interviews and case studies) to understand how best to re-specify and validate the typology (F_RQ6) is recommended Table 2. 
Table 2 Future research questions

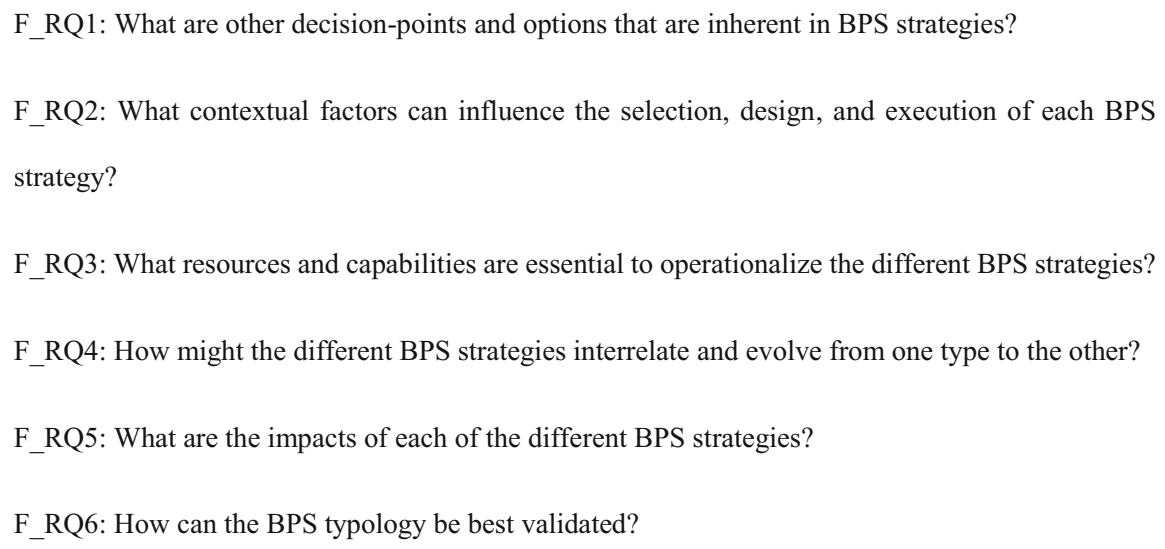

\section{Conclusion}

BPS is an emerging field (Afflerbach et al. 2016; Muenstermann 2015) and is gaining increased significance because of its application in many modern process related technologies, e.g., robotic process automation, process mining, and blockchain. However, BPS is still under-researched, with limited understanding of the BPS concept itself. We applied a multi-phased exploratory research approach to identify the different BPS strategies to contribute to addressing this conceptual gap.

Subsequent to a narrative and theoretical literature review, we used 21 cases from 18 BPS papers to understand the different strategies for Business Process Standardization (BPS). Content analysis of the relevant literature resulted in three decision- points: (D1) Approach, (D2) Type of Master Process, and (D3) Optimization of Master Process. The three decision-points and their options formed the basis of the proposed typology of 12 BPS strategies. These strategies were then instantiated using 21 published BPS cases. Of these 12 strategies, eight were instantiated by the cases, and the other four were explained using fictional case scenarios.

BPS is a significant and under-researched area, and the exploratory work presented in this paper, along with the proposed set of future research questions, provides concrete directions to develop further research in the area. The typology of BPS strategies presented in this paper provides a theory for analysis (Gregor 2006) and hence enables a better conceptual understanding of the concept of BPS. The typology also serves as a useful tool for researchers investigating the BPS concept and provides a lexicon that intends to help the discourse. It provides a framework to understand the different nuances of diverse BPS implementations and provides an appreciation of the different BPS strategic options.

This BPS typology is also of significance to practice. The study findings provide practitioners insight into the BPS process and the different decision-points and options. The description of the strategies and case examples for each strategy provides early insights regarding the organizational context(s) that suits different BPS strategies and how organizations can move from one strategy to another, which provides significant input to planning, implementation, and monitoring of BPS initiatives.

\section{References}

Afflerbach P, Bolsinger M, Röglinger M (2016) An economic decision model for determining the appropriate level of business process standardization. Bus Res 9(2):335-375. https://doi.org/ 10.1007/s40685-016-0035-6

Agnar G, Harry B, Mariano C (2004) The implementation process of standardisation. J Manuf Technol Manag 15(4):335-342. https:// doi.org/10.1108/17410380410535035

Aguirre S, Rodriguez A (2017) Automation of a business process using robotic process automation (RPA): a case study. In: Proceedings of the Workshop on Engineering Applications. https://doi.org/10.1007/978-3-319-66963-2_7

Asatiani A, Penttinen E (2016) Turning robotic process automation into commercial success - Case OpusCapita. J Inf Technol Teach Cases 6(2):67-74

AXELOS (2020) ITIL ${ }^{\circledR}$ Foundation Certification. https://www. axelos.com/certifications/itil-certifications/itil-foundation. Accessed 10 Oct 2020

Bandara W, Furtmueller E, Miskon S, Beekhuyzen J (2015) Achieving rigor in literature reviews: insights from qualitative data analysis and tool-support. Commun Assoc Inf Syst 34(8): 154-204

Becker M, Buchkremer R (2019) A practical process mining approach for compliance management. J Financ Regul Compl 27(4):464-478. https://doi.org/10.1108/JFRC-12-2018-0163 
Beimborn D, Gleisner F, Joachim N, Hackethal A (2009) The role of process standardization in achieving IT business value. In: Proceedings of the 42nd Hawaii International Conference on System Sciences. https://doi.org/10.1109/HICSS.2009.453

Bolstorff P, Rosenbaum RG (2007) Supply chain excellence: a handbook for dramatic improvement using the SCOR model. American Management Association, New York

Burattin A, van Zelst SJ, Armas-Cervantes A, van Dongen BF, Carmona J (2018) Online conformance checking using behavioural patterns. In: Proceedings of the International Conference on Business Process Management. https://doi.org/10.1007/9783-319-98648-7_15

Chinosi M, Trombetta A (2012) BPMN: an introduction to the standard. Comput Standards Interf 34(1):124-134

Collier D, LaPorte J, Seawright J (2012) Putting typologies to work: concept formation, measurement, and analytic rigor. Political Res Q 65(1):217-232

Dai Q, Kauffman RJ, Wang B (2011) The value of IT-enabled business process standardization from the real options perspective. In: Sharman R et al (eds) Exploring the Grand Challenges for Next Generation E-Business: 8th Workshop on E-Business, WEB 2009, Phoenix, Revised Selected Papers. Springer, Heidelberg, pp 160-165

Davenport TH, Short JE (1990) The new industrial engineering: information technology and business process redesign. Sloan Manag Rev 31(4):11-27

Doty DH, Glick WH (1994) Typologies as a unique form of theory building: toward improved understanding and modeling. Academy of Management. The Academy of Management Review 19(2):230

Dumas M, Rosa ML, Mendling J, Reijers HA (2013) Fundamentals of business process management. Springer, Heidelberg

Fereday J, Muir-Cochrane E (2008) Demonstrating rigor using thematic analysis: a hybrid approach of inductive and deductive coding and theme development. Int J Qual Methods 5(1):1-11

Fettke P, Loos P, Zwicker J (2005) Business process reference models: survey and classification. In: Proceedings of the Business Process Management Workshops. https://doi.org/10. 1007/11678564_44

Ganly D (2019) Deliver ERP Value by using both process standardization and best practices. Gartner, Stamford

García-Bañuelos L, Ponomarev A, Dumas M, Weber I (2017) optimized execution of business processes on blockchain. In: Carmona J et al (eds): Business Process Management. Springer, Cham. https://doi.org/https://doi.org/10.1007/978-3-319-650005

Goel $\bar{K}$ (2018) Potential role of business process standardization (BPS) on business correspondent (BC) model success. (Monograph), Queensland University of Technology, Brisbane

Goel K, Bandara W (2016) An understanding of business process standardization. In: Proceedings of the 30th Australian and New Zealand Academy of Management, Brisbane

Gottschalk F, van der Aalst WMP, Jansen-Vullers MH (2008) Mining reference process models and their configurations. In: Meersman $\mathrm{R}$ et al: Proceedings of the OTM Confederated International Conferences "On the Move to Meaningful Internet Systems". https://doi.org/10.1007/978-3-540-88875-8_47

Gregor S (2006) The nature of theory in information systems. MIS Q 30(3):611-642

Houy C, Fettke P, Loos P, van der Aalst WMP, Krogstie J (2011) Business process management in the large. Bus Inf Syst Eng 3(6):385-388. https://doi.org/10.1007/s12599-011-0181-5

ISO (1996) Standardization and related activities - General vocabulary. International Organization for Standardization, International Electrotechnical Commission, Geneva
Ji K, Pultz JE (2016) Optimizing I\&O costs in China. Gartner, Stamford

Kauffman RJ, Tsai JY (2010) With or without you: the countervailing forces and effects of process standardization. Electron Commer Res Appl 9(4):305-322. https://doi.org/10.1016/j.elerap.2009. 11.009

Kettenbohrer J, Beimborn D, Kloppenburg M (2013a) Developing a governance model for successful business process standardization. In: Proceedings of the 19th Americas Conference on Information Systems, Chicago. https://aisel.aisnet.org/ amcis2013/EndUserIS/GeneralPresentations/5/

Kettenbohrer J, Beimborn D, Kloppenburg M (2013b) Developing a procedural model for business process standardization. In: Proceedings of the 34th International Conference on Information Systems, Milano. https://aisel.aisnet.org/icis2013/proceedings/ ResearchInProgress/61/

Kwon S-W (2008) Does the standardization process matter? A study of cost effectiveness in hospital drug formularies. Manag Sci 54(6):1065-1079

Leshob A, Bourgouin A, Renard L (2018) Towards a process analysis approach to adopt robotic process automation. In: Proceedings of the IEEE 15th International Conference on e-Business Engineering. https://doi.org/10.1109/ICEBE.2018.00018

Li C, Reichert M, Wombacher A (2010) The MinAdept clustering approach for discovering reference process models out of process variants. Int J Coop Inf Syst 19(03n04):159-203

Manrodt KB, Vitasek K (2004) Glocal process standardization: a case study. J Bus Logist 25(1):1-23

Mendling J, Weber I, van der Aalst W et al (2018) Blockchains for business process management-challenges and opportunities. ACM Trans Manag Inf Syst 9(1):1-16

Microsoft (2008) Chevron drives process standardization and efficiency with mobile decision support. http://software.schneiderelectric.com/about-us/success-stories/listing-content/chevron/

Muenstermann B (2015) State of the art of BPS research business process standardization: a multi-methodological analysis of drivers and consequences. IGI Global, Hershey, pp 29-118

Muenstermann B, Eckhardt A (2009) What drives business process standardization? A case study approach. In: Proceedings of the International Conference on Information Resources Management. https://aisel.aisnet.org/cgi/viewcontent.cgi?article= $1046 \&$ context $=$ confirm 2009

Muenstermann B, Weitzel T (2008) What is process standardization? In: Proceedings of the International Conference on Information Resources Management. https://aisel.aisnet.org/confirm2008/64

Muenstermann B, Eckhardt A, Weitzel T (2009) Join the standard forces - Examining the combined impact of process and data standards on business process performance. In: Proceedings of the 42nd Hawaii International Conference on System Sciences. https://doi.org/10.1109/HICSS.2009.280

Muenstermann B, Eckhardt A, Weitzel T (2010) The performance impact of business process standardization. Bus Process Manag $\mathrm{J}$ 16(1):29-56. https://doi.org/10.1108/14637151011017930

Muenstermann B, Moederer P, Weitzel T (2010b) Setting up and managing business process standardization: insights from a case study with a multinational e-commerce firm. In: Proceedings of the 43rd Hawaii International Conference on System Sciences. https://doi.org/10.1109/HICSS.2010.477

Munoz-Gama J (2016) Conformance checking and diagnosis in process mining. Springer, Heidelberg

Narayanan S, Jayaraman V, Luo Y, Swaminathan JM (2011) The antecedents of process integration in business process outsourcing and its effect on firm performance. J Oper Manag 29(1-2):3-16. https://doi.org/10.1016/j.jom.2010.05.001 
Nesheim T (2011) Balancing process ownership and line management in a matrix-like organization. Knowl Process Manag 18(2):109-119. https://doi.org/10.1002/kpm.377

OMG (2011) Business process model and notation (BPMN) version 2.0. Object Management Group, OMG Document Number: formal/2011-01-03. http://www.omg.org/spec/BPMN/2.0

Paré G, Trudel M-C, Jaana M, Kitsiou S (2015) Synthesizing information systems knowledge: a typology of literature reviews. Inf Manag 52(2):183-199

Rahimi F, Møller C, Hvam L (2016) Succeeding in process standardization. Bus Process Manag J 22(6):1212-1246

Rehse J-R, Hake P, Fettke P, Loos P (2016) Inductive reference model development: recent results and current challenges. In: Mayr HC (ed) Informatik 2016, Springer, Heidelberg

Rehse J-R, Fettke P, Loos P (2017) A graph-theoretic method for the inductive development of reference process models. Softw Syst Modeling 16(3):833-873

Romano R (2004) The Sarbanes-Oxley Act and the making of quack corporate governance. Yale Law J 114:1521

Romero HL, Dijkman RM, Grefen PWPJ, van Weele AJ, de Jong A (2015) Measures of process harmonization. Inf Softw Technol 63:31-43. https://doi.org/10.1016/j.infsof.2015.03.004

Rosenkranz C, Seidel S, Mendling J, Schaefermeyer M, Recker J (2010) Towards a framework for business process standardization. In: Rinderle-Ma S et al (eds) Business Process Management Workshops, Ulm. Revised Papers, Springer, Heidelberg, pp 53-63

Roubert F, Beuzelin-Ollivier MG, Hofmann-Amtenbrink M, Hofmann H, Hool A (2016) "Nanostandardization" in action: implementing standardization processes in a multidisciplinary nanoparticle-based research and development project. Nanoethics 10(1):41-62. https://doi.org/10.1007/s11569-0150248-8

Sadiq S, Governatori G, Namiri K (2007) Modeling control objectives for business process compliance. In: Proceedings of the International conference on business process management. https:// doi.org/10.1007/978-3-540-75183-0_12

Saldana J (2012) The coding manual for qualitative researchers. Sage, Thousand Oaks

Schafermeyer M, Grgecic D, Rosenkranz C (2010) Factors influencing business process standardization: a multiple case study. In:
Proceedings of the 43rd Hawaii International Conference on System Sciences. https://doi.org/10.1109/HICSS.2010.207

Schäfermeyer M, Rosenkranz C, Holten R (2012) The Impact of business process complexity on business process standardization. Bus Inf Syst Eng 4(5):261-270

Scholta H, Niemann M, Delfmann P, Räckers M, Becker J (2019) Semi-automatic inductive construction of reference process models that represent best practices in public administrations: a method. Inf Syst 84:63-87

Seethamraju R, Seethamraju J (2009) Enterprise systems and business process agility - a case study. In: Proceedings of the $42 \mathrm{nd}$ Hawaii International Conference on System Sciences. https://doi. org/10.1109/HICSS.2009.196

Ungan MC (2006) Standardization through process documentation. Bus Process Manag J 12(2):135-148

van Wessel R, Ribbers P, de Vries H (2006, 04-07 Jan. 2006) Effects of IS standardization on business process performance: a case in HR IS company standardization. In: Proceedings of the 39th Annual Hawaii International Conference on System Sciences. https://doi.org/10.1109/HICSS.2006.141

vom Brocke J (2007) Design principles for reference modeling: reusing information models by means of aggregation, specialisation, instantiation, and analogy. In: Fettke P, Loos P (eds) Reference modeling for business systems analysis. IGI Global, pp 47-76

von Stetten A, Muenstermann B, Eckhardt A, Laumer S (2008) Towards an understanding of the business value of business process standardization - a case study approach. In: Proceedings AMCIS 2008, 20. https://aisel.aisnet.org/amcis2008/20

Winter R, vom Brocke J, Fettke P et al (2009) Patterns in business and information systems engineering. Bus Inf Syst Eng 1(6):468

Wullenweber K, Weitzel T (2007) An empirical exploration of how process standardization reduces outsourcing risks. In: Proceedings of the 40th Hawaii International Conference on System Sciences. https://doi.org/10.1109/HICSS.2007.63

Zellner P, Laumann M, Appelfeller W (2015) Towards managing business process variants within organizations - an action research study. In: Proceedings of the 48th Hawaii International Conference on System Sciences. doi: https://doi.org/10.1109/ HICSS.2015.495 